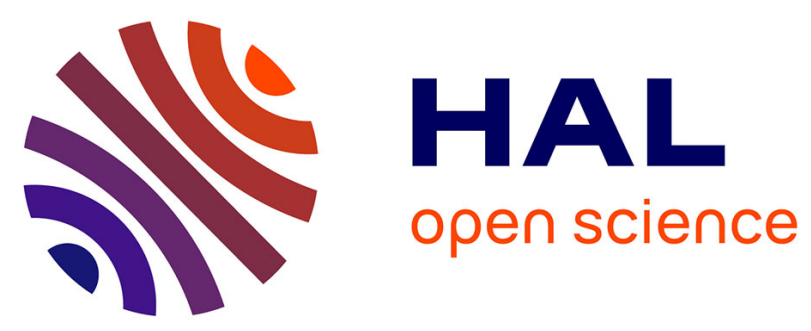

\title{
Using exponential time-varying gains for sampled-data stabilization and estimation
}

Tarek Ahmed-Ali, Emilia Fridman, Fouad Giri, Laurent Burlion, Francoise

Lamnabhi-Lagarrigue

\section{- To cite this version:}

Tarek Ahmed-Ali, Emilia Fridman, Fouad Giri, Laurent Burlion, Francoise Lamnabhi-Lagarrigue. Using exponential time-varying gains for sampled-data stabilization and estimation. Automatica, 2016, 67, pp.244-251. 10.1016/j.automatica.2016.01.048 . hal-01426572

\section{HAL Id: hal-01426572 \\ https://hal.science/hal-01426572}

Submitted on 5 Jul 2021

HAL is a multi-disciplinary open access archive for the deposit and dissemination of scientific research documents, whether they are published or not. The documents may come from teaching and research institutions in France or abroad, or from public or private research centers.
L'archive ouverte pluridisciplinaire $\mathbf{H A L}$, est destinée au dépôt et à la diffusion de documents scientifiques de niveau recherche, publiés ou non, émanant des établissements d'enseignement et de recherche français ou étrangers, des laboratoires publics ou privés. 


\title{
Using exponential time-varying gains for sampled-data stabilization and estimation
}

\author{
Tarek Ahmed-Ali a , Emilia Fridman ${ }^{\text {b }}$, Fouad Giri a , Laurent Burlion ${ }^{\text {c, }}$ \\ Françoise Lamnabhi-Lagarrigue ${ }^{\mathrm{d}}$ \\ a Laboratoire GREYC, UMR CNRS 6072, Université de Caen Basse-Normandie, ENSICAEN, Caen, France \\ ${ }^{\mathrm{b}}$ School of Electrical Engineering, Tel-Aviv University, Tel-Aviv 69978, Israel \\ ${ }^{\mathrm{c}}$ ONERA The French Aerospace Lab, 31055 Toulouse, France \\ d CNRS-INS2I, Laboratoire des Signaux et Systemes (L2S), European Embedded Control Institute, EECI Supelec, \\ 3 rue Joliot Curie, 91190 Gif-sur-Yvette, France
}

\begin{abstract}
This paper provides exponential stability results for two system classes. The first class includes a family of nonlinear ODE systems while the second consists of semi-linear parabolic PDEs. A common feature of both classes is that the systems they include involve sampled-data states and a time-varying gain. Sufficient conditions ensuring global exponential stability are established in terms of Linear Matrix Inequalities (LMIs) derived on the basis of Lyapunov-Krasovskii functionals. The established stability results prove to be useful in designing exponentially convergent observers based on sampled-data measurements. It is shown throughout simulated examples from the literature that the introduction of time-varying gains is beneficial to the enlargement of sampling intervals while preserving the stability of the system.
\end{abstract}

\section{Introduction}

Designing sampled-data observers and controllers has been a hot topic in recent years, see e.g. Fridman (2010) and reference list therein. In this regard, a long standing issue is how to enlarge the sampling intervals (Heemels, Johansson, \& Tabuada, 2012) while ensuring global exponential stability. In a recent paper (Cacace, Germani, \& Manes, 2014), it has been shown that the introduction of time-varying gains in a specific class of observers improves their exponential convergence properties in presence of measurement delay. Presently, these properties are investigated in presence of measurement sampling. To this end, we consider two classes of sampled-data systems and analyze their exponential stability. The

This work was partially supported by Israel Science Foundation (grant no. 1128/14). The material in this paper was partially presented at the 12th IFAC Workshop on Time Delay Systems, June 28-30, 2015, Ann Arbor, MI, USA (Ahmed-Ali, Fridman, Giri, Burlion, \& Lamnabhi-Lagarrigue, 2015). This paper was recommended for publication in revised form by Associate Editor Rafael Vazquez under the direction of Editor Miroslav Krstic.

E-mail addresses: tarek.ahmed-ali@ensicaen.fr (T. Ahmed-Ali), emilia@eng.tau.ac.il (E. Fridman), fouad.giri@unicaen.fr (F. Giri), Laurent.Burlion@onera.fr (L. Burlion), lamnabhi@lss.supelec.fr

(F. Lamnabhi-Lagarrigue). considered classes are respectively consisting of nonlinear globally Lipschitz ODEs and semi-linear parabolic PDEs. A common feature of both classes is that the systems they include are allowed to involve a time-varying gain of the form $e^{-\eta\left(t-t_{k}\right)}$ with $\eta>0$ a tuning parameter, where $t_{k}(k=0,1, \ldots)$ are sampling instants. It turns out that, the first family, including ODE systems, is a generalization of that dealt with in Cacace et al. (2014). For both classes of systems, we establish sufficient conditions for global exponential stability in terms of Linear Matrix Inequalities (LMIs) derived from Lyapunov-Krasovskii functionals. Then, it is shown that these stability results are useful in designing sampled-data observers with timevarying gains. As the established LMIs conditions involve both the tuning parameter $\eta$ and the maximum sampling interval $h$, these parameters can then be used to improve the observer convergence properties. Actually, it is checked through several simulated examples that the utilization of the above time-varying gain entails significant enlargement of the maximum sampling interval, compared with the constant gain case (corresponding to $\eta=0$ ). It is worth noting that, the present theoretical stability results can also be used in sampled-data control design improving exponential stability properties and enlarging sampling intervals. A part of the present results, namely those concerning ODEs, have been presented in our conference paper (Ahmed-Ali et al., 2015). 
The paper is organized as follows: in Section 2, the first stability result, concerning nonlinear ODE systems, is stated and applied to observer design; in Section 3, the second stability result, concerning semi-linear PDE systems, is stated and applied to observer design; a conclusion and reference list end the paper. Some technical proofs are appended.

\section{Notations and preliminaries}

Throughout the paper the superscript $T$ stands for matrix transposition, $\mathbf{R}^{n}$ denotes the $n$-dimensional Euclidean space with vector norm |.|, $\mathbf{R}^{n \times m}$ is the set of all $n \times m$ real matrices, and the notation $P>0$, for $P \in \mathbf{R}^{n \times n}$, means that $P$ is symmetric and positive definite. In Symmetric matrices, symmetric terms are denoted $* ; \lambda_{\min }(P)$ (resp. $\lambda_{\max }(P)$ ) denotes the smallest (resp. largest) eigenvalue. The notation $\left(t_{k}\right)_{k \geq 0}$ refers to a strictly increasing sequence such that $t_{0}=0$ and $\lim _{k \rightarrow \infty} t_{k}=\infty$. The sampling periods are bounded i.e. $0<t_{k+1}-t_{k}<h$ for some scalar $0<h<\infty$ and all $k=0,1, \ldots, \infty$. We also define the variable $\tau(t)=t-t_{k}, t \in\left[t_{k}, t_{k+1}\right) . \mathscr{H}^{1}(0, l)$ is the Sobolev space of absolutely continuous functions $z:(0, l) \rightarrow \mathbf{R}$ with the square integrable derivative $\frac{d}{d x}$. Given a two-argument function $u(x, t)$, its partial derivatives are denoted $u_{t}=\frac{\partial u}{\partial t}, u_{x x}=\frac{\partial^{2} u}{\partial x^{2}}$.

\section{Sampled-data globally Lipschitz nonlinear ODEs}

\subsection{System description and stability result}

We are considering a class of sampled-data nonlinear systems described by the following equation:

$\dot{x}(t)=A_{0} x(t)+A_{1} e^{-\eta\left(t-t_{k}\right)} x\left(t_{k}\right)+\phi(x(t)), \quad t \in\left[t_{k}, t_{k+1}\right)$

where $x(t) \in \mathbf{R}^{n}$; the scalar $\eta \geq 0 ; A_{0}, A_{1}$ are constant matrices with appropriate dimensions. As in Bar Am and Fridman (2014), the function $\phi$ is supposed to be class $\mathcal{C}^{1}$ with uniformly bounded Jacobian $\phi_{x}$, satisfying $\phi(0)=0$ and

$\phi_{x}^{T}(x) \phi_{x}(x) \leq M \quad \forall x$

for some positive constant $n \times n$-matrix $M$. Using Jensen's inequality it is readily checked that (2) implies the following inequality:

$\int_{0}^{1} \phi_{x}^{T}(s x) d s \int_{0}^{1} \phi_{x}(s x) d s \leq M$.

Remark 1. Eq. (1) may represent a networked control system described by

$\dot{x}(t)=A_{0} x(t)+\phi(x(t))+B u(t)$,

with the communication network placed between the sensor and the controller (but no network is placed between the controller and the actuator). Assuming that the discrete-time state measurements $x\left(t_{k}\right)$ are transmitted through the communication network from the sensor to controller, consider the state-feedback,

$u(t)=e^{-\eta\left(t-t_{k}\right)} K x\left(t_{k}\right), \quad t \in\left[t_{k}, t_{k+1}\right)$,

where $K$ is a gain and $\eta>0$ is a scalar. It turns out that the resulting closed-loop system fits Eq. (1) with $A_{1}=B K$.

As in Cacace et al. (2014), introduce the following change of coordinates $z(t)=e^{\eta t} x(t)$ with $\eta>0$. Then one gets

$$
\begin{aligned}
\dot{z}(t)= & \eta z(t)+A_{0} z(t)+A_{1} z\left(t_{k}\right) \\
& +\left[\int_{0}^{1} \phi_{x}(s x(t)) d s\right] e^{\eta t} x(t), \quad t \in\left[t_{k}, t_{k+1}\right)
\end{aligned}
$$

which is rewritten as follows:

$$
\begin{aligned}
\dot{z}(t)= & \left(\eta I_{n}+A_{0}\right) z(t)+A_{1} z\left(t_{k}\right) \\
& +\left[\int_{0}^{1} \phi_{x}(s x(t)) d s\right] z(t), \quad t \in\left[t_{k}, t_{k+1}\right) .
\end{aligned}
$$

Following Fridman (2010), consider the following LyapunovKrasovskii functional for (4):

$V(t)=\bar{V}(t)+V_{X}(t)$

with

$$
\begin{gathered}
\bar{V}(t)=z^{T}(t) P z(t)+\left(t_{k+1}-t\right) \int_{t_{k}}^{t} \dot{z}^{T}(s) U \dot{z}(s) d s, \\
P>0, U>0, t \in\left[t_{k}, t_{k+1}\right)
\end{gathered}
$$

and

$V_{X}(t)=\left(t_{k+1}-t\right) \xi^{T}\left[\begin{array}{cc}\frac{X+X^{T}}{2} & -X+X_{1} \\ * & -X_{1}-X_{1}^{T}+\frac{X+X^{T}}{2}\end{array}\right] \xi$,

where $\xi(t)=\operatorname{col}\left\{z(t), z\left(t_{k}\right)\right\}, X$ and $X_{1}$ are $n \times n$ matrices. The positiveness of $(5)$ is ensured if the following LMI holds (Fridman, 2010):

$$
\left[\begin{array}{cc}
P+h \frac{X+X^{T}}{2} & h X_{1}-h X \\
* & -h X_{1}-h X_{1}^{T}+h \frac{X+X^{T}}{2}
\end{array}\right]>0 .
$$

Using the definition of $z(t)$, we can see that the exponential stability of system (1) is guaranteed if:

$\dot{V}(t)+2 \alpha V(t) \leq 0 \quad t \in\left[t_{k}, t_{k+1}\right)$

for some scalar $\alpha \in(-\eta, 0]$ (note that the scalar $\alpha$ is allowed to be negative). Indeed, if ( 7 ) is satisfied one has,

$\dot{V}(t) \leq-2 \alpha V(t) \Longrightarrow|z(t)| \leq\left(\frac{\sqrt{V_{\mid t=0}}}{\sqrt{\lambda_{\min }(P)}}\right) e^{-\alpha t}$.

Then, using the fact that $z(t)=e^{\eta t} x(t)$, one gets:

$|x(t)| \leq\left(\frac{\sqrt{V_{\mid t=0}}}{\sqrt{\lambda_{\min }(P)}}\right) e^{-(\eta+\alpha) t}$.

From the above inequality, one sees that the exponential convergence is guaranteed if $\eta+\alpha>0$. Since the parameter $\eta$ is positive and free, it is sufficient to let $\alpha \in(-\eta, 0]$ for ensuring an exponential convergence with a decay rate $\eta+\alpha$. In the following proposition, it is shown that the property (7), and resulting exponential stability with a decay rate $\eta+\alpha>0$, are actually ensured under well established sufficient conditions, expressed in terms of LMIs.

Proposition 1. Consider the system (1) with possibly varying sampling-intervals subject to $t_{k+1}-t_{k} \leq h$ with some scalar $h>0$. Given $\eta>0$ and $\alpha \in(-\eta, 0]$, let there exist $n \times n$ matrices $P>0, U>0, X, X_{1}, P_{2}, P_{3}, T, Y_{1}, Y_{2}$ and a scalar $\lambda>0$ that satisfy the LMI (6) and the following LMIs:

$\Psi(0) \triangleq\left[\begin{array}{cccc}\Phi_{11}-X_{\alpha} & \Phi_{12}+X_{\tau(t)} & \Phi_{13}+X_{1 \alpha} & P_{2}^{T} \\ * & \Phi_{22}+h U & \Phi_{23}-X_{1 \tau(t)} & P_{3}^{T} \\ * & * & \Phi_{33}-X_{2 \alpha} & 0 \\ * & * & * & -\lambda I_{n}\end{array} \mid{ }_{\tau(t)=0}\right.$

$<0$ 
and

$$
\Psi(h) \triangleq\left[\left.\begin{array}{ccccc}
\Phi_{11}-X_{\alpha \mid} & \Phi_{12} & \Phi_{13}+X_{1 \alpha} & h Y_{1}^{T} & P_{2}^{T} \\
* & \Phi_{22} & \Phi_{23} & h Y_{2}^{T} & p_{3}^{T} \\
* & * & \Phi_{33}-X_{2 \alpha} & h T^{T} & 0 \\
* & * & * & -h U & 0 \\
* & * & * & * & -\lambda I_{n}
\end{array}\right|_{\tau(t)=h}\right.
$$$$
<0
$$

where

$$
\begin{aligned}
\Phi_{11}= & \left(A_{0}+\eta I_{n}\right)^{T} P_{2}+P_{2}^{T}\left(A_{0}+\eta I_{n}\right) \\
& +2 \alpha P-Y_{1}-Y_{1}^{T}+\lambda M \\
\Phi_{12}= & P-P_{2}^{T}+\left(A_{0}+\eta I_{n}\right)^{T} P_{3}-Y_{2} \\
\Phi_{13}= & Y_{1}^{T}+P_{2}^{T} A_{1}-T \\
\Phi_{22}= & -P_{3}-P_{3}^{T} \\
\Phi_{23}= & Y_{2}^{T}+P_{3}^{T} A_{1} \\
\Phi_{33}= & T+T^{T} \\
X_{\tau(t)}= & (h-\tau(t)) \frac{X+X^{T}}{2} \\
X_{\alpha}= & (1-2 \alpha(h-\tau(t))) \frac{X+X^{T}}{2} \\
X_{1 \tau(t)}= & (h-\tau(t))\left(X-X_{1}\right) \\
X_{1 \alpha}= & (1-2 \alpha(h-\tau(t)))\left(X-X_{1}\right) \\
X_{2 \alpha}= & (1-2 \alpha(h-\tau(t))) \frac{X+X^{T}-2 X_{1}-2 X_{1}^{T}}{2} .
\end{aligned}
$$

Then, the system (1) is exponentially stable with a decay rate $\eta+\alpha$. Moreover, if the above LMIs hold with $\alpha=-\eta$, then the system is exponentially stable with a small enough decay rate in $(0, \epsilon)$ for some $\epsilon \in(0,-\alpha)$.

See Appendix A for the proof.

Remark 2. Practically, the following procedure is used to get suitable values of the design parameters involved in Proposition 1. The search procedure is started by taking a small value of $\eta$ (e.g. $\eta=0)$. Then, one proceeds as follows:

(1) Let $\alpha=-\eta$

(2) Take a small value of $h$ and check the LMIs.

(3) Increase $h$ until the LMIs are no longer feasible. Then, retain the value of $h$.

(4) Increase the value of $\eta$ and repeat Steps (1)-(3).

(5) The above procedure is stopped when the increase of $\eta$ does not entail an increase of $h$.

Remark 3. Proposition 1 clearly shows that the maximum sampling interval $h$ that preserves the exponential stability is made dependent on the design parameters $\eta$ and $\alpha$. Additional highlights will be provided in Example 1 (given hereafter) where it is shown that the maximum $h$ preserving stability may be significantly enlarged by tuning $\eta$. On the other hand, Proposition 1 also confirms the somewhat obvious fact that the exponential decay rate $\eta+\alpha<\eta$ is also depending on $\eta$ and $\alpha$.

Remark 4. In the linear case,

$\dot{x}(t)=A_{0} x(t)+A_{1} e^{-\eta\left(t-t_{k}\right)} x\left(t_{k}\right), \quad t \in\left[t_{k}, t_{k+1}\right)$
Table 1

Max. values of $h$ vs. $\eta$ preserving the stability of Example 1.

\begin{tabular}{lllll}
\hline$\eta$ & 0 & 0.5 & 0.7 & 1 \\
$h$ & 1.38 & 1.84 & 2.36 & 3.17 \\
\hline
\end{tabular}

the function $\phi$ (see (1)) is zero and $M=0$ in (2). Then, it is sufficient to verify the feasibility of the smaller-order $\lambda$-independent LMIs (8) and (9) with the deleted last column and row. Indeed, the feasibility of the smaller-order LMIs implies the feasibility of the full-order LMIs with some $\lambda>0$.

Remark 5. If in (1) the matrix $A_{1}=B K$ depends on the unknown gain $K$, the inequalities (8) and (9) become nonlinear. However, by using the standard arguments for the controller design via the descriptor method (Fridman, 2014), where it is assumed that $P_{2}=\varepsilon P_{3}$ with a tuning parameter $\varepsilon$, one can easily derive LMIs for finding $K$.

\section{Example 1}

To illustrate the result of Proposition 1, consider the following system

$\dot{x}(t)=-x(t)+\sin (x(t))-K e^{-\eta\left(t-t_{k}\right)} x\left(t_{k}\right), \quad t \in\left[t_{k}, t_{k+1}\right)$

with $K=1$. Here we have $A_{0}=-1, A_{1}=-K=-1$, and $M=1$. Then, application of Proposition 1 with $\alpha=-\eta$ with various values of $\eta$ leads to different values of $h$ that preserve the exponential stability of the system (see Table 1). Thus, (10) with $\eta=1$ achieves exponential stability for more than twice larger sampling interval compared with the constant gain (i.e. case $\eta=0$ ). As a matter of fact, the parameter $\eta$ cannot be infinitely increased. Presently, the maximum value of $\eta$ that still yields an increase of the sampling interval is $\eta=1$.

\section{Example 2}

Consider the following much studied system (see e.g. Fridman, 2010, Zhang, Branicky, \& Phillips, 2001):

$\dot{x}(t)=\left[\begin{array}{cc}0 & 1 \\ 0 & -0.1\end{array}\right] x(t)+e^{-\eta\left(t-t_{k}\right)}\left[\begin{array}{c}0 \\ 0.1\end{array}\right] K x\left(t_{k}\right) \quad t \in\left[t_{k}, t_{k+1}\right)$

with $K=-[3.75,11.5]$. It is well-known that the above system with $\eta=0$ remains stable under constant sampling with $h<1.72$ and becomes unstable for constant sampling periods with $h>$ 1.73. Applying the result of Proposition 1, one obtains for $\eta=0.8$ and $\alpha=-0.8$ a maximum sampling interval value of $h=2.43$ that preserves the stability.

\section{Example 3}

Consider the following closed loop system:

$\dot{x}(t)=\left[\begin{array}{cc}0 & 1 \\ -1 & -2\end{array}\right] x(t)+\left[\begin{array}{l}0 \\ 1\end{array}\right] e^{-\eta\left(t-t_{k}\right)} K x\left(t_{k}\right) \quad t \in\left[t_{k}, t_{k+1}\right)$

with $K e^{-\eta\left(t-t_{k}\right)}=\left[\begin{array}{ll}-1 & 1\end{array}\right] e^{-\eta\left(t-t_{k}\right)}$ is the regulator gain. Applying the procedure of Remark 2 to system (11), one obtains the results of Table 2 . This shows that the time-varying nature of the regulator gain is actually beneficial to the enlargement of the sampling interval. The analysis of the closed loop system (11) in the case of a constant regulator gain (i.e. $\eta=0$ ) has been made in several previous studies using various approaches yielding different maximum sampling intervals. A comparison of the obtained maximum sampling intervals is made in Kao and $\mathrm{Wu}$ (2014), see Example 2 in Section 5 therein. The highest maximum sampling interval, obtained in Kao and Wu (2014), is $h=1.728$. Clearly, this value is much smaller than the maximum sampling interval achieved by using a time-varying regulator gain. 
Table 2

Max. values of $h$ vs. $\eta$ preserving the stability of Example 3.

\begin{tabular}{lllll}
\hline$\eta$ & 0.2 & 0.6 & 1 & 1.5 \\
$h$ & 2.03 & 3.38 & 4.69 & 5.60 \\
\hline
\end{tabular}

The above examples illustrate the fact that, when using a time varying gain, the maximum sampling interval $h$ that preserves the stability can be much larger compared to the constant gain case $(\eta=0)$.

\subsection{Application to sampled-data observer design}

Consider the class of nonlinear systems

$\left\{\begin{array}{l}\dot{x}(t)=A x(t)+f(x(t)) \\ y(t)=C x(t)\end{array}\right.$

where $x \in \mathbf{R}^{n}$ and $A, C$ are matrices with appropriate dimensions. It is supposed that the function $f$ satisfies assumption (2) and $y$ is accessible to measurements only at instants $t_{k}$. We also suppose that the pair $(A, C)$ is detectable. The following observer is then proposed:

$$
\begin{aligned}
& \dot{\hat{x}}(t)=A \hat{x}(t)+f(\hat{x}(t))-K e^{-\eta\left(t-t_{k}\right)}\left(C \hat{x}\left(t_{k}\right)-y\left(t_{k}\right)\right) \\
& \quad t \in\left[t_{k}, t_{k+1}\right),
\end{aligned}
$$

with $\eta>0$ and $K$ is a matrix gain of appropriate dimension. Our goal is to determine a matrix $K$ and a maximum sampling period $h$ so that, the observation error $\tilde{x}(t)=\hat{x}(t)-x(t)$ is globally exponentially stable. It is readily checked that this error satisfies the following equation:

$$
\begin{aligned}
\dot{\tilde{x}}(t)= & A \tilde{x}(t)+f(\hat{x}(t))-f(x(t)) \\
& -K e^{-\eta\left(t-t_{k}\right)}\left(C \hat{x}\left(t_{k}\right)-y\left(t_{k}\right)\right) \quad t \in\left[t_{k}, t_{k+1}\right) .
\end{aligned}
$$

Considering the change of coordinates $z_{1}(t)=e^{\eta t} \tilde{x}(t)$, one gets the following equation

$$
\dot{z}_{1}(t)=\left(\eta I_{n}+A\right) z_{1}(t)-K C z_{1}\left(t_{k}\right)+e^{\eta t}(f(\hat{x}(t))-f(x(t))) .
$$

Using the fact that,

$f(\hat{x}(t))-f(x(t))=\left[\int_{0}^{1} f_{x}(x(t)+s(\hat{x}(t)-x(t))) d s\right] \tilde{x}(t)$,

Eq. (14) can be rewritten as follows:

$$
\begin{aligned}
& \dot{z}_{1}(t)=\left(\eta I_{n}+A\right) z_{1}(t)+A_{1} z_{1}\left(t_{k}\right) \\
& \quad+\left[\int_{0}^{1} f_{x}(x(t)+s(\hat{x}(t)-x(t))) d s\right] z_{1}(t), \quad t \in\left[t_{k}, t_{k+1}\right)
\end{aligned}
$$

with $A_{1}=-K C$. Clearly, Eq. (15) falls in the class of systems described by Eq. (4). Therefore, the result of Proposition 1 can directly be applied to get sufficient conditions for the observer (13) to be exponentially convergent. This is illustrated in the next example.

\section{Example 4}

Consider the following nonlinear system

$$
\left\{\begin{array}{l}
\dot{x}_{1}(t)=x_{2}(t) \\
\dot{x}_{2}(t)=-2 x_{1}(t)-x_{2}(t)+0.2 \sin \left(x_{2}(t)\right) \\
y(t)=C x(t)=x_{1}(t)
\end{array}\right.
$$

Clearly, this system is of the form (12) with $A=\left(\begin{array}{cc}0 & 1 \\ -2 & -1\end{array}\right), C=$ $(1,0)$, and $f(x(t))=\left(\begin{array}{ll}0 & 0.2 \sin \left(x_{2}(t)\right)\end{array}\right)^{T}$.
Table 3

Max. values of $h$ vs. $\eta$ preserving the stability of Example 4.

$\begin{array}{lllll}\eta & 0 & 1 & 2 & 2.4 \\ h & 0.99 & 1.64 & 2.78 & 2.86\end{array}$

It is readily checked that the function $f$ satisfies inequality (2) with $M=\operatorname{diag}\{0,0.04\}$. Then the observer (13) is written as follows:

$\left\{\begin{array}{l}\dot{\hat{x}}_{1}(t)=\hat{x}_{2}(t)-K^{1} e^{-\eta\left(t-t_{k}\right)}\left(\hat{x}_{1}\left(t_{k}\right)-y\left(t_{k}\right)\right) \\ \dot{\hat{x}}_{2}(t)=-2 \hat{x}_{1}(t)-\hat{x}_{2}(t)+0.2 \sin \left(\hat{x}_{2}(t)\right) \\ -K^{2} e^{-\eta\left(t-t_{k}\right)}\left(\hat{x}_{1}\left(t_{k}\right)-y\left(t_{k}\right)\right)\end{array}\right.$

where $K=\left[K_{1} K_{2}\right]$ and $A_{1}=-K C=\left(\begin{array}{ll}-K^{1} & 0 \\ -K^{2} & 0\end{array}\right)$. Applying Proposition 1 with $K=[1.65,-0.85]^{T}$ and $\alpha=-\eta$, one obtains the results of Table 3 .

This clearly shows that the maximum sampling interval $h$ can be significantly increased thanks to the time-varying gain feature of the observer.

Remark 6. Note that high gain observers are particular cases of the class of observers described by (13). They correspond to $K=$ $\theta \Delta^{-1} L$ where $\theta \geq 1, \Delta=\operatorname{diag}\left\{1, \ldots, 1 / \theta^{n-1}\right\}$ and $L$ is such that $A-L C$ is Hurwitz. Then the function in (12) must be triangular (Gauthier, Hammouri, \& Othman, 1992). Compared to the timevarying gain observer proposed in Farza et al. (2014), our observer (13) enjoys a simpler time-varying gain (because it is scalar) and offers more flexibility because the parameter $\eta$ is independent on the gain $K$. In this respect, one might also note that the timeinvariant-gain version of the observer proposed in Farza et al. (2014) is nothing other than an obvious rewriting in impulsive form of the original observer of Karafyllis and Kravaris (2009).

\section{Sampled-data semi-linear parabolic PDEs}

\subsection{System description and stability result}

In this section, it is shown that results similar to those of Section 2 can be established for infinite-dimensional systems. Let us consider the class of systems governed by the following partial differential equation:

$$
\begin{aligned}
u_{t}(x, t)= & u_{x x}(x, t)+\phi(x, u(x, t), t) u(x, t) \\
& -K e^{-\eta\left(t-t_{k}\right)} u\left(\bar{x}_{j}, t_{k}\right) \quad t \in\left[t_{k}, t_{k+1}\right), x \in\left[x_{j}, x_{j+1}\right),
\end{aligned}
$$

with $\bar{x}_{j}=\frac{x_{j+1}+x_{j}}{2}(j=0, \ldots, N-1)$, where the points $x_{j}$ divide the interval $[0, l]$ such that $0=x_{0}<\cdots<x_{N}=l$, under Neumann boundary conditions

$u_{x}(l, t)=u_{x}(0, t)=0, \quad t \geq 0$.

We also suppose that the function $\phi$ is $\mathcal{C}^{1}$ and is possibly unknown but $\phi_{m} \leq \phi \leq \phi_{M}$, where $\phi_{m}$ and $\phi_{M}$ are known bounds. The sampling interval in space may be variable but bounded

$x_{j+1}-x_{j} \leq \Delta \leq l$.

By arguments developed in Fridman and Blighovsky (2012), there exists a unique strong solution of (16) initialized by

$u(\cdot, 0) \in \mathscr{H}^{1}(0, l): u_{x}(0,0)=u_{x}(l, 0)=0$.

Proposition 2. Consider the class of systems described by (16) and the positive scalars $\eta>0, h>0, R>0,2 \eta>\delta_{1}>0,2 \delta \in$ $\left(\delta_{1}-2 \eta, 0\right]$. Choose $K>\phi_{M}+\eta-\frac{\pi^{2}}{l^{2}}$ and suppose that there exist scalars $p_{i}>0, r>0$, and $y_{i}(i=1,2,3)$, satisfying the inequality

$\Delta K R^{-1}\left(p_{2}+p_{3}\right) \leq \pi \delta_{1} p_{3}$ 
and the following LMIs:

$\Phi_{\mid \phi=\phi_{m}}^{i}<0, \quad \Phi_{\mid \phi=\phi_{M}}^{i}<0, \quad i=0,1$

where

$\Phi^{0} \triangleq\left[\begin{array}{ccc}\chi_{11}-\lambda & \chi_{12} & \chi_{13} \\ * & h r+\chi_{22} & \chi_{23} \\ * & * & \chi_{33}\end{array}\right]$

$\Phi^{1} \triangleq\left[\begin{array}{cccc}\chi_{11}-\lambda & \chi_{12} & \chi_{13} & h y_{1} \\ * & \chi_{22} & \chi_{23} & h y_{2} \\ * & * & \chi_{33} & h y_{3} \\ * & * & * & -h r\end{array}\right]$

with

$\chi_{11}=2 \delta p_{1}+2 p_{2}\left(\phi+\eta+\frac{\Delta K R}{2 \pi}\right)-2 y_{1}$

$\chi_{12}=p_{1}-p_{2}+p_{3}(\phi+\eta)-y_{2}$

$\chi_{13}=y_{1}-y_{3}-K p_{2}$

$\chi_{22}=-2 p_{3}+\frac{\Delta K R p_{3}}{\pi}$

$\chi_{23}=y_{2}-K p_{3}$

$\lambda=2 \frac{\pi^{2}}{l^{2}}\left(p_{2}-\delta p_{3}\right)$

$\chi_{33}=2 y_{3}-\delta_{1} p_{1}$.

Then, the unique strong solution of the system (16) initiated by (18) satisfies the following bound, for all $t \geq 0$ :

$$
\begin{aligned}
& \int_{0}^{l}\left[p_{1} u^{2}(x, t)+p_{3} u_{x}^{2}(x, t)\right] d x \\
& \quad \leq e^{\left(-2(\delta+\eta)+\delta_{1}\right) t} \int_{0}^{l}\left[p_{1} u^{2}(x, 0)+p_{3} u_{x}^{2}(x, 0)\right] d x,
\end{aligned}
$$

accordingly the system (16) is exponentially stable with a decay rate $(\delta+\eta)-\delta_{1} / 2$. Moreover, if the LMIs (20) hold with $2 \delta=\delta_{1}-2 \eta$, then the system (16) is exponentially stable with a decay rate in $(0, \epsilon)$ for some $\epsilon \in\left(0, \delta_{1}\right)$.

See the proof placed in Appendix B.

Remark 7. A practical selection procedure similar to the ODE case (Remark 2) is used to find out suitable values of the design parameters of Proposition 2. Again, the search is started by considering a small value of $\eta$, (e.g. $\eta=0$ ).

(1) Choose $\delta_{1}$ arbitrarily in the interval $(0,2 \eta)$ and let $\delta=\delta_{1} / 2-\eta$.

(2) Take a small value of $h$ and check the LMIs.

(3) Increase $h$ until the LMIs are no longer feasible. Then, retain the value of $h$.

(4) Increase the value of $\eta$ and repeat Steps (1)-(3).

(5) The above procedure is stopped when the increase of $\eta$ entails no increase of $h$.

\section{Example 5}

Consider the following system:

$$
\begin{gathered}
u_{t}(x, t)=u_{x x}(x, t)-K e^{-\eta\left(t-t_{k}\right)} u\left(\bar{x}_{j}, t_{k}\right) \\
x \in\left[x_{j}, x_{j+1}\right), t \in\left[t_{k}, t_{k+1}\right)
\end{gathered}
$$

under the Neumann boundary conditions. This system fits system structure (16), with $\phi(x, u)=0$. Applying Proposition 2 with $l=\pi, K=1, \Delta=\pi / 7, R=1$, one gets for different values of $\eta$ the maximum sampling interval that preserves the exponential stability. The results thus obtained are summarized in Table 4 which shows that the maximum sampling interval $h$ can be substantially enlarged by tuning $\eta$.
Table 4

Max.values of $h$ vs. $\eta$ that preserve the stability of Example 5.

\begin{tabular}{lllccc}
\hline$\eta$ & 0 & 0.25 & 0.75 & 1 & 2 \\
$\delta_{1}$ & 0.5 & 0.5 & 0.5 & 0.5 & 0.5 \\
$\delta$ & 0.25 & 0 & -0.25 & -0.5 & -0.75 \\
$h$ & 1.27 & 1.95 & 1.8 & 1.73 & 1.41 \\
\hline
\end{tabular}

\subsection{Application to sampled-data observer design}

Consider the following semi-linear diffusion equation:

$u_{t}(x, t)=u_{x x}(x, t)+f(u(x, t), x, t)$

with Neumann condition $u_{x}(0,0)=u_{x}(l, 0)=0$. The system output, $y\left(t_{k}\right)=u\left(\bar{x}_{j}, t_{k}\right)$, for some $j$, is only available at sampling instants $t_{k}$. It is supposed that the function $f$ is known, of class $\mathcal{C}^{1}$, and satisfying $f_{m} \leq f_{u} \leq f_{M}$, for some scalar constants $f_{m}$ and $f_{M}$. The following observer structure is considered:

$$
\begin{gathered}
\hat{u}_{t}(x, t)=\hat{u}_{x x}(x, t)+f(\hat{u}(x, t), x, t) \\
-L e^{-\eta\left(t-t_{k}\right)}\left(\hat{u}\left(\bar{x}_{j}, t_{k}\right)-y\left(t_{k}\right)\right) \\
x \in\left[\bar{x}_{j}, \bar{x}_{j+1}\right), t \in\left[t_{k}, t_{k+1}\right)
\end{gathered}
$$

with $\hat{u}_{x}(0, t)=\hat{u}_{x}(l, t)=0$. It is readily checked that the observation error $e(x, t)=\hat{u}(x, t)-u(x, t)$ undergoes the following equation:

$$
\begin{aligned}
e_{t}(x, t)= & e_{x x}(x, t)+\phi(e(x, t), x, t) e(x, t) \\
& -L e^{-\eta\left(t-t_{k}\right)} e\left(\bar{x}_{j}, t_{k}\right), \quad x \in\left[\bar{x}_{j}, \bar{x}_{j+1}\right), t \in\left[t_{k}, t_{k+1}\right)
\end{aligned}
$$

where $\phi=\int_{0}^{l} f_{u}(\hat{u}+\theta e, x, t) d \theta$, with boundary conditions $e_{x}(0, t)=e_{x}(l, t)=0$. Clearly, Eq. (24) is of the form (16). Therefore, one can make use of Proposition 2 to determine the observer gain $L$ as well as a suitable value of the maximum $h$, while guaranteeing the exponential convergence of the observer (23).

\subsection{Example 6}

Heat/mass transfer systems with heat generation or volumetric chemical reactions, e.g. chemical tubular reactor, are examples of systems that can be described by (16), see Boskovic and Krstic (2002) and references therein. Indeed, such systems undergo a heat equation with non-constant coefficient i.e.

$u_{t}=\epsilon_{u} u_{x x}+\lambda_{\alpha \beta}(x) u(x, t), \quad x \in(0,1)$

with $u_{x}(0, t)=u_{x}(1, t)=0$, where

$\lambda_{\alpha \beta}(x)=\frac{2}{\cosh ^{2}(\alpha x-\beta)}$.

Let $\epsilon_{u}=1, \alpha=4$ and $\beta=2$ and consider the following observer:

$\hat{u}_{t}=\hat{u}_{x x}+\lambda_{\alpha \beta}(x) \hat{u}(x, t)$

$$
-L e^{-\eta\left(t-t_{k}\right)}\left(\hat{u}\left(\bar{x}_{j}, t_{k}\right)-y\left(t_{k}\right)\right), \quad x \in(0,1)
$$

with $\hat{u}_{x}(0, t)=\hat{u}_{x}(1, t)=0$. It is readily checked that the observation error $e(x, t)=\hat{u}(x, t)-u(x, t)$ undergoes Eq. (24) with $\phi(e, x, t)=\phi(x)=\lambda_{\alpha \beta}(x)$ and the boundary conditions $e_{x}(0, t)=e_{x}(1, t)=0$. It can be shown that one presently has $\phi_{M}=30$ and $\phi_{m}=0$. Applying the above procedure with $R=1, \Delta=0.001, L=0.11+\phi_{M}+\eta-\pi^{2}$, several possible sampling interval values, corresponding to different values of the parameter $\eta$, have been obtained, see Table 5 . It is readily seen that the observer time-varying gain associated with $\eta=2$ provides a maximum sampling interval that is nearly 10 times larger than the case of fixed observer gain (i.e. case $\eta=0$ ). 
Table 5

Max. values of $h$ vs. $\eta$ that preserve the stability of Example 6 .

\begin{tabular}{llrccc}
\hline$\eta$ & 0 & 0.5 & 0.75 & 1 & 2 \\
$\delta_{1}$ & 0.5 & 0.5 & 0.5 & 0.5 & 0.5 \\
$\delta$ & 0.25 & -0.25 & -0.5 & -0.75 & -1.75 \\
$h \times 10^{3}$ & 3 & 16.5 & 19.9 & 22.6 & 29 \\
\hline
\end{tabular}

\section{Conclusion}

Novel stability results, stated in Propositions 1 and 2, are established for two classes of systems respectively described by ODEs and PDEs. Both studied classes involve the exponentially decaying term $e^{-\eta\left(t-t_{k}\right)}$ and all stability conditions are expressed in terms of LMIs. The new results conditions are shown to be useful in designing sampled-data observers with exponentially decaying gains. Furthermore, it is checked through several numerical examples that the introduction of exponentially decaying gains may lead to a substantial enlargement of the maximum sampling intervals while preserving stability. A theoretical proof that, timevarying gain entails sampling interval enlargement is yet to be found. Finally, the extension of the results presented in the present paper, to the case of disturbances is a topic for our future research.

\section{Appendix A. Proof of Proposition 1}

Let $\alpha \in(-\eta, 0]$ and differentiate $V(t)$. After some simple computations one obtains:

$$
\begin{aligned}
& \dot{V}(t)+2 \alpha V(t)+\lambda z^{T}(t) \\
& \times\left(M-\int_{0}^{1} \phi_{x}^{T}(s x) d s \int_{0}^{1} \phi_{x}(s x) d s\right) z(t) \\
& \leq 2 z^{T}(t) P \dot{z}(t)+\left(t_{k+1}-t\right) \dot{z}^{T}(t) U \dot{z}(t)-\int_{t_{k}}^{t} \dot{z}^{T}(s) U \dot{z}(s) d s \\
& -\xi^{T}\left[\begin{array}{cc}
\frac{X+X^{T}}{2} & -X+X_{1} \\
* & -X_{1}-X_{1}^{T}+\frac{X+X^{T}}{2}
\end{array}\right] \xi \\
& +\left(t_{k+1}-t\right)\left[\dot{z}^{T}(t)\left(X+X^{T}\right) z(t)\right. \\
& \left.+2 \dot{z}^{T}(t)\left(-X+X_{1}\right) z\left(t_{k}\right)\right]+2 \alpha z^{T}(t) P z(t) \\
& +2 \alpha\left(t_{k+1}-t\right) \xi^{T}\left[\begin{array}{cc}
\frac{X+X^{T}}{2} & -X+X_{1} \\
* & -X_{1}-X_{1}^{T}+\frac{X+X^{T}}{2}
\end{array}\right] \xi \\
& +\lambda z^{T}(t) M z(t) \\
& -\lambda z^{T}(t)\left(\int_{0}^{1} \phi_{x}^{T}(s x) d s \int_{0}^{1} \phi_{x}(s x) d s\right) z(t)
\end{aligned}
$$

using the well known Jensen's inequality, one gets,

$\int_{t_{k}}^{t} \dot{z}^{T}(s) U \dot{z}(s) d s \geq\left(t-t_{k}\right) v_{1}^{T} U v_{1}$

with $v_{1}=\frac{1}{t-t_{k}} \int_{t_{k}}^{t} \dot{z}^{T}(s)$. According to the descriptor approach (Fridman, 2001), the left-hand sides of the equations,

$$
\begin{aligned}
0= & 2\left[z^{T}(t) Y_{1}^{T}+\dot{z}^{T}(t) Y_{2}^{T}+z^{T}\left(t_{k}\right) T^{T}\right] \\
& \times\left[-z(t)+z\left(t_{k}\right)+\left(t-t_{k}\right) v_{1}\right]
\end{aligned}
$$

$$
\begin{aligned}
0= & 2\left[z^{T}(t) P_{2}^{T}+\dot{z}^{T}(t) P_{3}^{T}\right] \\
\times & {\left[\left(A_{0}+\eta I_{n}\right) z(t)+A_{1} z\left(t_{k}\right)+\left(\int_{0}^{1} \phi_{x}(s x) d s\right) z(t)-\dot{z}\right] }
\end{aligned}
$$

can be added to $\dot{V}(t)+2 \alpha V(t)$ where $Y_{1}, Y_{2}, T, P_{2}, P_{3}$ are free matrices. Let us define the augmented vector,

$\mu=\operatorname{col}\left\{z(t), \dot{z}(t), z\left(t_{k}\right), v_{1},\left(\int_{0}^{1} \phi_{x}(s x) d s\right) z(t)\right\}$.

Then, combining (A.2) and (A.1), one gets:

$$
\begin{aligned}
& \dot{V}(t)+2 \alpha V(t)+\lambda z^{T}(t)\left(M-\int_{0}^{1} \phi_{x}^{T}(s x) d s \int_{0}^{1} \phi_{x}(s x) d s\right) z(t) \\
& \quad \leq \mu^{T} \Psi \mu<0
\end{aligned}
$$

provided that

$\Psi \triangleq\left[\begin{array}{ccccc}\Phi_{11}-X_{\alpha} & \Phi_{12}+X_{\tau(t)} & \Phi_{13}+X_{1 \alpha} & \tau(t) Y_{1}^{T} & P_{2}^{T} \\ * & \Phi_{22}+s . U & \Phi_{23}-X_{1 \tau(t)} & \tau(t) Y_{2}^{T} & p_{3}^{T} \\ * & * & \Phi_{33}-X_{2 \alpha} & \tau(t) T^{T} & 0 \\ * & * & * & -\tau(t) U & 0 \\ * & * & * & * & -\lambda I_{n}\end{array}\right]$

$<0$.

with $s=t_{k+1}-t$. Writing the last matrix inequality for $\tau(t) \rightarrow 0$ and $\tau(t) \rightarrow h$ leads to LMIs (8) and (9), respectively. Now, using arguments of Lemma 2 in Fridman (2010), it is not difficult to see that, if the LMIs (6), (8) and (9) are feasible for some $h>0$, then they are also feasible for all $\bar{h} \in(0, h]$.

Now, consider the case where $\alpha=-\eta$. If the LMIs (6), (8) and (9) are feasible, then by continuity, they are also feasible with the same $\eta$ and $\bar{\alpha}=-\eta+\epsilon$ for small enough $\epsilon>0$. Then, an inequality like (A.3) holds with $\alpha$ being replaced by $\bar{\alpha}$. Then, one gets

$\dot{V}(t)+\bar{\alpha} V \leq 0$

which yields

$|x(t)| \leq\left(\frac{\sqrt{V_{\mid t=0}}}{\sqrt{\lambda_{\min }(P)}}\right) e^{-(\eta+\bar{\alpha}) t}=\left(\frac{\sqrt{V_{\mid t=0}}}{\sqrt{\lambda_{\min }(P)}}\right) e^{-\epsilon t}$.

This completes the proof of Proposition 1.

\section{Appendix B. Sketch of proof of Proposition 2}

Let us introduce the change of coordinates $\xi(x, t)=e^{\eta t} u(x, t)$ with $\eta>0$. Then it is checked that $\xi(x, t)$ undergoes the equation:

$$
\begin{aligned}
\xi_{t}(x, t)= & \xi_{x x}(x, t)+(\phi(x, u(x, t), t)+\eta) \xi(x, t) \\
& -K \xi\left(\bar{x}_{j}, t_{k}\right) \quad t \in\left[t_{k}, t_{k+1}\right), x \in\left[\bar{x}_{j}, \bar{x}_{j+1}\right) .
\end{aligned}
$$

Consider the following Lyapunov-functional inspired by Fridman and Blighovsky (2012):

$$
\begin{aligned}
V_{1}(t)= & p_{1} \int_{0}^{l} \xi(x, t)^{2} d x+\int_{0}^{l}\left[p_{3} \xi_{x}^{2}(x, t)+r\left(t_{k+1}-t\right)\right. \\
& \left.\times \int_{t_{k}}^{t} e^{2 \delta(s-t)} z_{s}^{2}(x, s) d s\right] d x, \quad t \in\left[t_{k}, t_{k+1}\right) .
\end{aligned}
$$

Notice that the above functional is continuous i.e. it satisfies $V_{1}\left(t_{k}\right)=V_{1}\left(t_{k}^{-}\right)$. The rest of the proof matches mutatis mutandis 
a similar proof in Fridman and Blighovsky (2012). Relying on the descriptor technique, the two following equalities are considered:

$$
\begin{aligned}
0= & 2 \int_{0}^{l}\left[p_{2} \xi(x, t)+p_{3} \xi_{t}(x, t)\right] \times\left[-\xi_{t}(x, t)\right. \\
& \left.+\xi_{x x}(x, t)+(\phi(x, u(x, t))+\eta) \xi(x, t)-K \xi\left(x, t_{k}\right)\right] d x \\
& +2 K \sum_{j=1}^{N-1} \int_{x_{j}}^{x_{j+1}}\left[p_{2} \xi(x, t)+p_{3} \xi_{t}(x, t)\right] \\
& \times \int_{\bar{x}_{j}}^{x} \xi_{\zeta}\left(\zeta, t_{k}\right) d \zeta d x \\
0= & 2 \int_{0}^{l}\left[y_{1} \xi(x, t)+y_{2} \xi_{t}(x, t)+y_{3} \xi\left(x, t_{k}\right)\right] \\
& \times\left[-\xi(x, t)+\xi\left(x, t_{k}\right)+\left(t-t_{k}\right) v_{2}(x, t)\right] d x
\end{aligned}
$$

with

$v_{2}=\frac{1}{t-t_{k}} \int_{t_{k}}^{t} \xi_{s}(x, s) d s$

where the first equality is directly got from (B.1) while the second is obtained using Leibnitz-Newton formula. Adding the right sides of equalities (B.2), (B.3) to $\dot{V}_{1}(t)+2 \delta V_{1}(t)-\delta_{1} V_{1}\left(t_{k}\right)$, one obtains after some computations the following inequality:

$$
\begin{aligned}
& \dot{V}_{1}(t)+2 \delta V_{1}(t)-\delta_{1} V_{1}\left(t_{k}\right) \leq \int_{0}^{l} \eta_{2}^{T} \bar{\Phi}_{s} \eta_{2} d x \\
& +\left[\frac{\Delta}{\pi} K R^{-1}\left(p_{2}+p_{3}\right)-\delta_{1} p_{3}\right] \int_{0}^{l} \xi_{x}^{2}\left(x, t_{k}\right) d x
\end{aligned}
$$

with

$$
\eta_{2}=\operatorname{col}\left\{\xi(x, t), \xi_{t}(x, t), \xi\left(x, t_{k}\right), v_{2}\right\}
$$

and

$\bar{\Phi}_{s}=\left[\begin{array}{cccc}\chi_{11} & \chi_{12} & \chi_{13} & \left(t-t_{k}\right) y_{1} \\ * & \left(t_{k+1}-t\right) r+\chi_{22} & \chi_{23} & \left(t-t_{k}\right) y_{2} \\ * & * & \chi_{33} & \left(t-t_{k}\right) y_{3} \\ * & * & * & -\left(t-t_{k}\right)\end{array}\right]$.

Just as in Fridman and Blighovsky (2012), it can be seen that if the LMIs (20) are feasible, then $\bar{\Phi}_{s} \leq 0$. If further (19) is fulfilled, then the following inequality holds:

$\dot{V}_{1}(t)+2 \delta V_{1}(t)-\delta_{1} V_{1}\left(t_{k}\right) \leq 0 \quad t \in\left[t_{k}, t_{k+1}\right)$.

Since $2 \delta<0$, one easily gets

$\left|\dot{V}_{1}(t)\right| \leq\left(-2 \delta+\delta_{1}\right) \sup _{t_{k} \leq s<t}\left(V_{1}(s)\right) \quad t \in\left[t_{k}, t_{k+1}\right)$.

Now, let us define the following continuous functional:

$\psi(t)=\sup _{t_{k} \leq s<t}\left(V_{1}(s)\right), \quad t \in\left[t_{k}, t_{k+1}\right)$.

It is readily checked that

$\lim _{h \rightarrow 0^{+}}\left(\sup \frac{\psi(t+h)-\psi(t)}{h}\right) \leq\left(-2 \delta+\delta_{1}\right) \psi(t)$.

Then, by using the comparison Lemma 2.12 in Karafyllis and Jiang (2011), one obtains

$V_{1}(t) \leq \sup _{t_{k} \leq s<t}\left(V_{1}(s)\right) \leq V_{1}(0) e^{\left(-2 \delta+\delta_{1}\right) t}, \quad t \geq 0$.

This, together with the following inequality

$$
\begin{aligned}
& \int_{0}^{l} e^{2 \eta t}\left[p_{1} u^{2}(x, t)+p_{3} u_{x}^{2}(x, t)\right] d x \leq V_{1}(t) \\
& \quad \leq\left[\int_{0}^{l}\left[p_{1} u^{2}(x, 0)+p_{3} u_{x}^{2}(x, 0)\right] d x\right] e^{\left(-2 \delta+\delta_{1}\right) t}, \quad t \geq 0
\end{aligned}
$$

yields (21).
Now, consider the case where $\delta_{1}=2 \delta+2 \eta$. If the LMIs (20) are feasible, then by continuity, they are also feasible with the same $\eta$ and $\bar{\delta}_{1}=2 \delta+2 \eta-\epsilon$ for small enough $\epsilon>0$. Then, an inequality like (B.4) holds with $\delta_{1}$ being replaced by $\bar{\delta}_{1}$. Then, one gets

$V_{1}(t) \leq \sup _{t_{k} \leq s<t}\left(V_{1}(s)\right) \leq V_{1}(0) e^{\left(-2 \delta+\bar{\delta}_{1}\right) t}, \quad t \geq 0$

which yields

$\int_{0}^{l}\left[p_{1} u^{2}(x, t)+p_{3} u_{x}^{2}(x, t)\right] d x \leq V_{1}(0) e^{-\epsilon t}$.

This completes the proof of Proposition 2.

\section{References}

Ahmed-Ali, T., Fridman, E., Giri, F., Burlion, L., \& Lamnabhi-Lagarrigue, F. (2015). A new approach to enlarging sampling intervals for some sampled-data systems and observers. In 12th IFAC workshop on time delay systems. USA: University of Michigan.

Bar Am, N., \& Fridman, E. (2014). Network-based distributed $h_{\infty}$-filtering of parabolic systems. Automatica, 50, 3139-3146.

Boskovic, D., \& Krstic, M. (2002). Backstepping control of chemical tubular reactor. Computers and Chemical Engineering, 1077-1085.

Cacace, F., Germani, A., \& Manes, C. (2014). Nonlinear systems with multiple timevarying measurement delays. SIAM Journal on Control and Optimization, 52(3), $1862-1885$.

Farza, M., M'saad, M., Fall, M. L., Pigeon, E., Gehan, O., \& Busawon, K. (2014) Continuous-discrete time observers for a class of mimo nonlinear systems. IEEE Transactions on Automatic Control, 59(4), 1060-1065.

Fridman, E. (2001). New Lyapunov Krasovskii functionals for stability of linear retarded and neutral type systems. Systems E' Control Letters, 43(4), 309-319.

Fridman, E. (2010). A refined input delay approach to sampled-data control. Automatica, 46, 421-427.

Fridman, E. (2014). Systems and control: foundations and applications, Introduction to time-delay systems: analysis and control. Birkhauser.

Fridman, E., \& Blighovsky, A. (2012). Robust sampled-data control of a class of semilinear parabolic systems. Automatica, 48, 826-836.

Gauthier, J. P., Hammouri, H., \& Othman, S. (1992). A simple observer for nonlinear systems applications to bioreactors. IEEE Transactions on Automatic Control, 37(6), 875-880.

Heemels, W.P.M.H., Johansson, K.H., \& Tabuada, P. (2012). An introduction to eventtriggered and self-triggered control. In IEEE conference on decision and control vol. 46 (pp. 3270-3285).

Kao, C.Y., \& Wu, D.R. (2014). On robust stability of aperiodic sampled-data systemsan integral quadratic constraint approach. In IEEE American control conference, Portland, Oregon, USA (pp. 4871-4876).

Karafyllis, I., \& Jiang, Z.-P. (2011). Stability and stabilization of nonlinear systems (1st ed.). New York: Springer Verlag.

Karafyllis, I., \& Kravaris, C. (2009). From continuous-time design to sampleddata design of nonlinear observers. IEEE Transactions on Automatic Control, 54, 2169-2174.

Zhang, W., Branicky, M., \& Phillips, S. (2001). Stability of networked control systems. Automatica, 21, 84-99. 